\title{
Effects Of Candesartan Cilexetil Drug And Allium Sativum On Certain Enzymes And Biochemical Parameters In Hyperlipidemic Mice
}

\author{
Mahmoud R. Mahmoud \\ Zoology Department, Faculty Of Science, Zagazig University, Egypt
}

\begin{abstract}
The present study was performed to investigate the possible correlation between the effects of candesartan cilexetil (3 mg/kg b.w.) and garlic; allium sativum (100 mg/kg b.w.) alone and in combination on high cholesterol diet (HCD) male mice . Candesartan cilexetil alone and their combination with garlic were given orally to animals fed on hypercholesterolemic diet by gavage for 12 weeks . Results of this investigation showed that animals under high cholesterol diet exhibited some sort of changes in the blood, plasma and tissue levels of leukocytes,cholesterol, triglyceride and enzymes where they increased significantly while some decrease exhibited in heart weight, LDH-cholesterol and testosterone hormone compared with that of the normal diet animals. Candesartan cilexetil garlic or their combination significantly decreased the plasma and tissue levels of total cholesterol (TC), triglyceride (TG), while they increased significantly the levels of high density lipoprotein cholesterol ( HDL-C). On the contrary, candesartan and candesartan in combination with garlic decrease WBCs, neutrophils and platelets significantly in compared with that of the (HCD) animals. This study included the total body and heart weights. Heart weight increased significantly in candesartan, garlic and their combination treated group. On the other hand, there were significantly decreases in body weight in candesartan, garlic and their combination compared with that of the high cholesterol diet treated animals. The effect of candesartan, garlic and their combination upon serum and liver levels of ALP, AST, ALT and ChE enzymes were investigated. Serum and liver ALP, AST, ALT and ChE were increased significantly in candesartan treated group but they decreased markedly in garlic and its combination with candesartan in serum and liver respectively compared with that of the high cholesterol diet animals. Candesartan decreased plasma level of testosterone while garlic and its combination with candesartan increased the testosterone hormone significantly compared with that of the high cholesterol diet animals. In conclusion, animals given garlic have improved physiological functions and induced significant causes of antiatherosclerosis effect. Also, these observations show that garlic is efficacious in lowering blood lipid profils and thereby could maintain protection with hypertensive drugs.
\end{abstract}

\section{Introduction}

A number of prospective studies have established that the risk of cardiac morbidity and mortality is directly related to the concentration of plasma cholesterol (Kannel et al.,1971). Despite the development of a number of agents that effectively reduce serum cholesterol levels in patients, coronary arteriosclerosis and subsequent myocardial infraction still represent a major health concern in many studies (Wesley et al.,1999). Many studies investigating the effects of myocardial ischemia-reperfusion in the setting of hypercholesterolemia have focused primarly on rabbit models of ischemia alone or on ischemia-reperfusion (Golino et al., 1987; Tilton et al.,1987). Osborne et al. (1987) reported that creatin kinase release after 5 hours of regional ischemia was significantly greater in rabbits fed a high 
cholesterol diet for 10 to 12 weeks. Furthermore, a subsequent study by Osborne et al.(1989) of myocardial infarction in hypercholesterolemic rabbits reported an increased of myocardial tissue injury that was significantly reversed by treatment with the 3-hydroxy-3-methylglutaryl coenzyme A reductase inhibitor lovastatin. It was also reported by Golino et al.(1987) that platelets depletion markedly reduced myocardial infarction size and extent of no-reflow in rabbits fed a $2.0 \%$ cholesterol diet for 3 days. Myocardial infarction, the major complications of hypertension and atherosclerotic vascular disease continue to be the important public health problem in developed nations ( Neal and MacMahon,1999). Chobanian and Alexander (1996) suggested that high blood pressure may have a direct role in enhancing atherosclerotic lesion formation. The available evidence indicated that hypertension appears to induce a sequence of changes in the connective tissue metabolism, smooth muscle cells , endothelial integrity, platelet function, lipid profile and change pertaining to insulin and glucose metabolism which may be important in the genesis of atherosclerosis in hypertensive subjects (Doyle,1991 and Weber et al.,1991). Clinically, many antihypertensive drugs are effective in reducing morbidity and mortility from atherosclerotically mediated cardiovascular events. As hypertension is a long -term disorder however one of the most perplexing problems in management of hypertension is the adverse effect of antihypertension drugs or their metabolite on cardiovascular system. The effect may be small but can blunt considerably the beneficial effect of blood pressure reduction (Takishita,2001).

Tissue catecholamines were studied in spontaneously hypertension rats that were long-term treated with candesartan. Noradrenaline and adrenaline contents were doubled in the left ventricle but not in liver or cortex. In parallel, cardiac monoamine oxidase activity was reduced significantly by candesartan (Raasch et al.,2002). Moran (2002) indicated that the combination of candesartan to angiotensin converting enzyme inhibitor (ACE I) may offer additional protection in preventing progression in chronic renal disease in patients. Garlic and its preparation have been recognized as agent for prevention and treatment of cardiovascular and other metabolic disease, atherosclerosis, hyperlipidemia, thrombosis, hypertension and diabetes (Banerjee and Maulik,2002). The clinical research by Spigelski and Jones (2001) suggested that consumption of garlic powder does not play a significant role in lowering plasma lipid levels when in conjunction with a low fat, low cholesterol diet. Garlic powder caused a significant dose dependent relaxation in intralobar pulmonary arterial rings both in the presence and absence of nitric oxide synthase blockers and intact endothelium (Fallon et al.,1998). Berthold et al.(1998) investigated that the commertial garlic oil preparation had no influence on serum lipoproteins, cholesterol absorption or cholesterol synthesis in humans. The use of garlic and garlic preparations as agents for prevention and treatment of atherosclerosis and atherosclerosis-related diseases. Garlic indirectly effects atherosclerosis by reduction of hyperlipidemia, hypertension and probably diabetes mellitus and prevents thrombus formation. In addition, in animal models, garlic causes direct antiatherogenic and antiatherosclerotic effects at the level of artery wall (Agarwal,1996 ; Orekhov and Grunwald,1997).

The present investigation was undertaken to find out the possible influence of angiotensin II receptor blocker (candesartan cilexetil) and garlic powder on the development of atherosclerosis and their side effects in cholesterol fed animals.

\section{Material And Methods}

Twenty five male mice weighing 27-35 g. were divided into four groups ( 5 animals in each group). After one week of initial adaptation to animal chew. Each group of animals was treated for 12 weeks as the follows:

Group1 : Control given 5\% gum arabic solution alone daily without atherogenic diet for 12 weeks. . 
Group2: Animals were fed 10 g. of atherogenic diet $(50 \mathrm{mg}$ cholesterol and 9.5 $\mathrm{g}$ chew) daily for 12 weeks.

Group3: Candesartan cilexetil (Takeda Chemical Industries Ltd.,AstraZeneca) with atherogenic diet daily for 12 weeks.

Group4: Garlic (ATOS PharmaCairo/Egypt) $100 \mathrm{mg} / \mathrm{kg}$ with atherogenic diet daily for 12 weeks.

Candesartan cilexelit and garlic were administered by gavage one hour before atherogenic diet. For gavage administration, candesartan and garlic solution were prepared daily by mixing each drug powder with 5\% gum Arabic in double distilled water. The mixtures were vortexed for 5 min and administered by gavage daily for 12 weeks. Control animals were given 5\% gum arabic solution alone daily without atherogenic diet for 12 weeks.

Blood samples were collected in heparinized tubes after decapitation of animals after overnight fasting at the end of 12 weeks. The collected blood samples were used for hematological and blood film study (Hawk et al.,1965). The remaining blood was centrifuged at $4000 \mathrm{rpm}$ for $10 \mathrm{~min}$. and one part of plasma was used for determination of biochemical studies and hormonal assays.

Alkaline phosphatase enzyme (ALP) was setermined according to Belfield and Goldberg (1971). Aspartate Aminotransferase (AST) and Alanine Aminotransferase (ALT) enzymes were determined according to Reitman and Frankel (1975). Acetylcholinesterase enzyme (AChE) was determined according to Elman et al. (1979). Plasma levels of total cholesterol and triglycerides were measured colorimetrically using the Bio-analytical kits according to Buccolo and David (1973) . High density lipoprotein cholesterol (HDLc) levels were measured according to method of Warnick et al (1983). The plasma levels of testosterone was determined by radioimmunoassay (RIA) using commertial kits from Cis Bio International, France according to Velez et al.(1988). The results obtained in this study were statistically analyzed using student t'test according to Snedecor and Cochran (1969).

\section{Results}

Table (1) lists the total cholesterol, triglyceride, HDL-c and testosterone hormone in plasma and tissues in normal diet (ND) and high cholesterol diet (HCD) treated animals. Total cholesterol and triglyceride were increased significantly from $11 \%$ to $86 \%$ in plasma, aortic, liver and adrenal respectively in high cholesterol diet compared with that of normal diet animals. Also, HDL-c and testosterone plasma levels were decreased markedly (49\% and 8\%) in high cholesterol diet compared with that of the normal diet animals. Total WBCs, neutrophils and platelets were significantly increased $(37 \%$ to $152 \%$ ) while serum and liver enzyme levels of ALP, AST, ALT and AChE had no significant effect between two groups (Tables 2 and 3). Table (3) indicated that the heart weight decreased $(21 \%)$ accompanied with increase $(21 \%)$ of body weight significantly. Levels of plasma total cholesterol, triglyceride showed $24 \%$ to $59 \%$ significant decrease in candesartan, garlic and their combination compared with that of the high cholesterol diet animals while candesartan, garlic and their combination treated mice showed from $156 \%$ to $224 \%$ markedly increase of HDL-c compared with that of the high cholesterol diet animals (Table 4). On the other hand, tissue cholesterol levels showed high significant decrease (18\% to 61\%) in candesartan, garlic and their combination compared with high cholesterol diet treated animals (Table 5). Total WBCs, neutrophils and platelets recorded highly signifycant decrease in animals treated with candesartan, garlic and their combination ( $22 \%$ to $62 \%$ ) compared with high cholesterol diet animals (Table 6). Heart weight recorded significant increase ( $41 \%$ and $4 \%$ ) in candesartan and its combination with garlic accompainent by significant decrease (25\% and $30 \%)$ in candesartan and garlic groups compared with that of the high cholesterol diet animals (Table 7). Table (8) shows a notable decrease in plasma testosterone hormone in candesartan treated animals (18\%) while it shows a marked increase in garlic and its 
combination with candesartan treated animals (44\% and 34\%) compared with that of the high cholesterol diet treated animals. Tables (9 and 10) show that candesartan caused a significant increases in serum and liver enzymes (from $15 \%$ to $115 \%$ ) but garlic treated animals recorded a significant decrease from $10 \%$ to $64 \%$ while the combination of candesartan and garlic show significant increase $(64 \%$ and $71 \%)$ in serum accompanied with significant decrease ( from $28 \%$ to $36 \%$ ) in liver compared with that of high cholesterol diet animals.

Table (1): Plasma levels of total cholesterol, triglyceride, high density lipoprotein cholesterol (mmol/L), tissue cholesterol contents ( $\mathrm{mg} / \mathrm{g}$ wet weight ) and plasma testosterone (ug/ml) levels of normal diet (ND) and cholesterol diet (HCD) of male mice. Mean \pm S.E.of five animals.

\begin{tabular}{|l|l|l|l|l|l|l|l|}
\hline $\begin{array}{l}\text { Paramete } \\
\text { rs }\end{array}$ & $\begin{array}{l}\text { Total } \\
\text { cholestero } \\
\mathbf{l}\end{array}$ & $\begin{array}{l}\text { Ttrigl- } \\
\text { yceride }\end{array}$ & HDL-c & $\begin{array}{l}\text { Aortic } \\
\text { cholester } \\
\text { ol }\end{array}$ & $\begin{array}{l}\text { Liver } \\
\text { cholesterol }\end{array}$ & $\begin{array}{l}\text { Adrenal } \\
\text { cholesterol }\end{array}$ & $\begin{array}{l}\text { Testo- } \\
\text { sterone }\end{array}$ \\
\hline ND & $11.98 \pm$ & $1.46 \pm$ & $0.50 \pm$ & $7.0 \pm$ & $24.42 \pm$ & $53.8 \pm$ & $1.06 \pm$ \\
& 0.19 & 0.06 & 0.017 & 0.28 & 1.81 & 1.65 & 0.04 \\
\hline HCD & $13.32 \pm$ & $1.78 \pm$ & $0.25 \pm$ & $13.4 \pm$ & $35.42 \pm$ & $95.8 \pm$ & $0.89 \pm$ \\
& $0.33^{* *}$ & $0.08 * *$ & $0.013^{* * *}$ & $0.41 * * *$ & $1.31^{* * *}$ & $1.46^{* * *}$ & $0.045^{*}$ \\
\hline \%change & $+11.2 \%$ & $+21.9 \%$ & $-49.2 \%$ & $+86.3 \%$ & $+45.0 \%$ & $+78.1 \%$ & $-7.5 \%$ \\
\hline
\end{tabular}

*Significant at $\mathrm{P}<0.05, * *$ high significant at $\mathrm{P}<0.01$, ***very highly significant at $\mathrm{P}<$ 0.001 respectively.

Table (2): Total WBCs $x 10^{3} / \mu \mathrm{l}$, Neutrophils $\times 10^{3} / \mu$ l, Platelets $\times 10^{5} / \mu \mathrm{l}$ and serum enzyme levels of normal (ND) and cholesterol diet $(\mathrm{HCD})$ of male mice. Mean \pm S.E. of five animals.

\begin{tabular}{|c|l|l|l|l|l|l|c|}
\hline $\begin{array}{l}\text { Paramete } \\
\text { rs }\end{array}$ & $\begin{array}{l}\text { Total } \\
\text { WBCs }\end{array}$ & Neutrophil & Platelets & $\begin{array}{l}\text { ALP } \\
(\mathbf{U} / \mathbf{L})\end{array}$ & $\begin{array}{l}\text { AST } \\
(\mathbf{U} / \mathbf{m l})\end{array}$ & $\begin{array}{l}\text { ALT } \\
(\mathbf{U} / \mathbf{m l})\end{array}$ & $\begin{array}{c}\text { AChE } \\
(\mathbf{U} / \mathbf{L})\end{array}$ \\
\hline ND & $2.42 \pm$ & $1.16 \pm$ & $9.84 \pm$ & $52.84 \pm$ & $95.8 \pm$ & $65.6 \pm$ & $349.2 \pm$ \\
& 0.06 & 0.05 & 0.17 & 1.92 & 0.82 & 1.07 & 19.17 \\
\hline HCD & $5.48 \pm$ & $2.92 \pm$ & $13.5 \pm$ & $53.4 \pm$ & $100.1 \pm$ & $66.32 \pm$ & $359.0 \pm$ \\
& $0.14 * * *$ & $0.13 * * *$ & $0.20 * * *$ & $1.92^{\text {n.s. }}$ & $2.31^{\text {n.s. }}$ & $2.54^{\text {n.s. }}$ & $19.77^{\text {n.s. }}$ \\
\hline \%change & $+126.4 \%$ & $+151.7 \%$ & $+37.2 \%$ & $+1.1 \%$ & $+4.5 \%$ & $+1.1 \%$ & $+2.1 \%$ \\
\hline
\end{tabular}

*Significant at $\mathrm{P}<0.05$, ** high significant at $\mathrm{P}<0.01$, ***very highly significant at $\mathrm{P}<$ 0.001 respectively.

Table (3): The heart and body weights (g.) and liver enzyme levels of normal (ND) and high cholesterol diet $($ HCD) male mice. Mean \pm S.E. of five animals.

\begin{tabular}{|l|l|l|l|l|l|l|}
\hline parameters & $\begin{array}{l}\text { Heart } \\
\text { weight }\end{array}$ & $\begin{array}{l}\text { Body } \\
\text { weight }\end{array}$ & $\begin{array}{l}\text { ALP } \\
(\mathbf{U} / \mathbf{L})\end{array}$ & $\begin{array}{l}\text { AST } \\
(\mathbf{U} / \mathbf{m l})\end{array}$ & $\begin{array}{l}\text { ALT } \\
(\mathbf{U} / \mathbf{m l})\end{array}$ & $\begin{array}{l}\text { AChE } \\
(\mathbf{U} / \mathbf{L})\end{array}$ \\
\hline ND & $0.198 \pm$ & $22.8 \pm$ & $11.74 \pm$ & $56.42 \pm$ & $14.72 \pm$ & $47.36 \pm$ \\
& 0.01 & 0.37 & 0.34 & 1.11 & 0.22 & 1.16 \\
\hline HCD & $0.156 \pm$ & $27.6 \pm$ & $12.94 \pm$ & $51.98 \pm$ & $15.32 \pm$ & $45.56 \pm$ \\
& $0.004 * * *$ & $0.68^{* * *}$ & $0.45^{\text {n.s. }}$ & $2.36^{\text {n.s. }}$ & $0.29^{\text {n.s. }}$ & $1.58^{\text {n.s. }}$ \\
\hline \%change & $-21.2 \%$ & $+21.1 \%$ & $+10.2 \%$ & $-7.9 \%$ & $+4.1 \%$ & $-3.8^{\%}$ \\
\hline
\end{tabular}

*Significant at $\mathrm{P}<0.05$, ** high significant at $\mathrm{P}<0.01$, ***very highly significant at $\mathrm{P}<$ 0.001 respectively. 
Table (4): Effects of candesartan( $3 \mathrm{mg} / \mathrm{kg}$ body weight), garlic $(100 \mathrm{mg} / \mathrm{kg})$ and their combination on plasma levels of total cholesterol, triglyceride, high density lipoprotein cholesterol (mmol/L) compared with cholesterol diet (HCD) treated male mice. Mean \pm S.E.of five animals.

\begin{tabular}{|c|c|c|c|c|}
\hline parameters & HCD & Candesartan & Garlic & $\begin{array}{l}\text { Candesartan } \\
\text { + Garlic }\end{array}$ \\
\hline $\begin{array}{l}\text { Total cholesterol } \\
\text { (Mean } \pm \text { S.E.) }\end{array}$ & $\begin{array}{l}13.32 \pm \\
0.33\end{array}$ & $\begin{array}{l}10.14 \pm \\
0.32 * * *\end{array}$ & $\begin{array}{l}8.24 \pm \\
0.33 * * *\end{array}$ & $\begin{array}{l}7.94 \pm \\
0.48 * * *\end{array}$ \\
\hline \% Change & & $-24.2 \%$ & $-41.8 \%$ & $-40.7 \%$ \\
\hline $\begin{array}{l}\text { Triglyceride } \\
(\text { Mean } \pm \text { S.E) }\end{array}$ & $\begin{array}{l}1.78 \pm \\
0.08\end{array}$ & $\begin{array}{l}1.03 \pm \\
0.08 * * *\end{array}$ & $\begin{array}{c}0.83 \pm \\
0.03 * * *\end{array}$ & $\begin{array}{l}0.72 \pm \\
0.03 * * *\end{array}$ \\
\hline \% Change & & $-42.0 \%$ & $-53.4 \%$ & $-59.6 \%$ \\
\hline $\begin{array}{l}\text { HDL-C } \\
(\text { Mean } \pm \text { S.E) }\end{array}$ & $\begin{array}{l}0.25 \pm \\
0.013\end{array}$ & $\begin{array}{l}0.67 \pm \\
0.011^{* * *}\end{array}$ & \multirow{2}{*}{$\begin{array}{l}0.81 \pm \\
0.04 * * * \\
+224.0 \%\end{array}$} & \multirow{2}{*}{$\begin{array}{l}0.64 \pm \\
0.03 * * * \\
+156.0 \%\end{array}$} \\
\hline \% Change & & $+168.0 \%$ & & \\
\hline
\end{tabular}

*Significant at $\mathrm{P}<0.05, * *$ high significant at $\mathrm{P}<0.01, * * *$ very highly significant at $\mathrm{P}<$ 0.001 respectively.

Table (5): Effects of candesartan(3 mg/kg body weight), garlic $(100 \mathrm{mg} / \mathrm{kg})$ and their combination on tissue cholesterol contents (mg/g wet weight of tissue ) compared with cholesterol diet (HCD) treated male mice. Mean \pm S.E.of five animals.

\begin{tabular}{|c|c|c|c|c|}
\hline Parameters & HCD & Candesartan & Garlic & $\begin{array}{l}\text { Candesartan } \\
\text { + Garlic }\end{array}$ \\
\hline $\begin{array}{l}\text { Aortic cholesterol } \\
(\text { Mean } \pm \text { S.E. })\end{array}$ & $\begin{array}{l}13.4 \pm \\
0.41\end{array}$ & $10.1 \pm 0.38 * * *$ & $\begin{array}{l}5.8 \pm \\
0.25^{*}\end{array}$ & $\begin{array}{l}6.6 \pm \\
0.48^{\text {n.s }}\end{array}$ \\
\hline \% Change & & $-22.2 \%$ & $-55.3 \%$ & $-50.7 \%$ \\
\hline $\begin{array}{l}\text { Liver cholesterol } \\
(\text { Mean } \pm \text { S.E) }\end{array}$ & $35.42 \pm 1.31$ & $\begin{array}{l}23.06 \pm \\
1.65^{\text {n.s. }}\end{array}$ & $\begin{array}{l}20.6 \pm \\
0.24 *\end{array}$ & $\begin{array}{l}17.6 \pm \\
0.81 * *\end{array}$ \\
\hline$\%$ Change & & $-34.8 \%$ & $-41.8 \%$ & $-50.3 \%$ \\
\hline $\begin{array}{l}\text { Adrenal cholesterol } \\
\text { (Mean } \pm \text { S.E) }\end{array}$ & $95.8 \pm 1.46$ & $65.2 \pm 3.2 * *$ & \multirow{2}{*}{$\begin{array}{l}44.6 \pm \\
1.96 * * \\
-53.4 \%\end{array}$} & \multirow{2}{*}{$\begin{array}{l}38.8 \pm \\
1.16 * * * \\
-61.2 \%\end{array}$} \\
\hline \% Change & & $-31.9 \%$ & & \\
\hline
\end{tabular}

*Significant at $\mathrm{P}<0.05$, ** high significant at $\mathrm{P}<0.01$, ***very highly significant at $\mathrm{P}<$ 0.001 respectively. 
Table (6):Effects of candesartan $(3 \mathrm{mg} / \mathrm{kg}$ body weight), garlic $(100 \mathrm{mg} / \mathrm{kg})$ and their combination on total WBCs $\times 10^{3} / \mu \mathrm{l}$, Neutrophils $\times 10^{3} / \mu \mathrm{l}$ and Platelets $\times 10^{5} / \mu \mathrm{l}$ of compared with cholesterol diet $(\mathrm{HCD})$ treated male mice. Mean \pm S.E. of five animals.

\begin{tabular}{|c|c|c|c|c|}
\hline Parameters & HCD & Candesartan & Garlic & $\begin{array}{l}\text { Candesartan } \\
\text { + Garlic }\end{array}$ \\
\hline $\begin{array}{l}\text { Total WBCs } \\
(\text { Mean } \pm \text { S.E. })\end{array}$ & $\begin{array}{l}5.48 \pm \\
0.14\end{array}$ & $\begin{array}{l}2.68 \pm \\
0.28^{* * * *}\end{array}$ & $\begin{array}{l}2.40 \pm \\
0.18^{* * * *}\end{array}$ & $\begin{array}{c}2.08 \pm \\
012^{*}\end{array}$ \\
\hline \% Change & & $-51.1 \%$ & $-56.2 \%$ & $-62.0 \%$ \\
\hline $\begin{array}{l}\text { Neutrophil } \\
(\text { Mean } \pm \text { S.E) }\end{array}$ & $\begin{array}{l}2.92 \pm \\
0.13\end{array}$ & $\begin{array}{l}2.26 \pm \\
0.14^{* *}\end{array}$ & $\begin{array}{l}0.88 \pm \\
0.05^{*}\end{array}$ & $\begin{array}{l}1.36 \pm \\
0.17^{\text {n.s. }}\end{array}$ \\
\hline \% Change & & $-22.6 \%$ & $-69.9 \%$ & $-53.4 \%$ \\
\hline $\begin{array}{l}\text { Platelets } \\
(\text { Mean } \pm \text { S.E) }\end{array}$ & $\begin{array}{l}13.5 \pm \\
0.20\end{array}$ & $\begin{array}{l}8.68 \pm^{* * *} \\
0.71^{* *}\end{array}$ & $\begin{array}{c}8.84 \pm \\
0.41 * * *\end{array}$ & $\begin{array}{c}7.98 \pm \\
0.27 * * *\end{array}$ \\
\hline \% Change & & $-35.7 \%$ & $-34.5 \%$ & $-40.9 \%$ \\
\hline
\end{tabular}

n.s. denoted to non significant, ${ }^{*}$ Significant at $\mathrm{P}<0.05, * *$ high significant at $\mathrm{P}<0.01$, $* * *$ very highly significant at $\mathrm{P}<0.001$ respectively.

Table (7):Effects of candesartan(3 mg/kg body weight), garlic $(100 \mathrm{mg} / \mathrm{kg})$ and their combination on the heart and body weights (g.) compared with cholesterol diet (HCD) treated male mice. Mean \pm S.E.of five animals.

\begin{tabular}{|l|l|l|l|l|}
\hline Parameters & HCD & Candesartan & Garlic & $\begin{array}{l}\text { Candesartan } \\
\text { + Garlic }\end{array}$ \\
\hline Heart weight & $0.156 \pm$ & $0.22 \pm$ & $0.19 \pm$ & $0.162 \pm$ \\
Mean \pm S.E. & 0.004 & $0.004 *$ & $0.005^{\text {n.s. }}$ & $0.006^{* *}$ \\
\hline \% Change & & $+41.0 \%$ & $+21.8 \%$ & $+3.8 \%$ \\
\hline Body weight & $27.6 \pm$ & $20.8 \pm$ & $19.4 \pm$ & $22.0 \pm$ \\
Mean \pm S.E. & 0.68 & $0.37 * *$ & $0.75 * *$ & $0.71^{\text {n.s. }}$ \\
\hline \% Change & & $-24.6 \%$ & $-29.7 \%$ & $-22.5 \%$ \\
\hline
\end{tabular}

n.s. denoted to nonsignificant, *Significant at $\mathrm{P}<0.05$, ** high significant at $\mathrm{P}<0.01$, $* * *$ very highly significant at $\mathrm{P}<0.001$ respectively.

Table (8): Effects of candesartan $(3 \mathrm{mg} / \mathrm{kg}$ body weight), garlic $(100 \mathrm{mg} / \mathrm{kg})$ and their combination on plasma testosterone $(\mathrm{ug} / \mathrm{ml})$ levels compared with cholesterol diet (HCD) treated male mice. Mean \pm S.E. of five animals.

\begin{tabular}{|l|l|l|l|l|}
\hline Parameters & HCD & Candesartan & Garlic & $\begin{array}{l}\text { Candesartan } \\
\text { + Garlic }\end{array}$ \\
\hline $\begin{array}{l}\text { Testosterone } \\
\text { (Mean } \pm \text { S.E.) }\end{array}$ & $0.89 \pm 0.05^{*}$ & $0.73 \pm$ & $1.28 \pm$ & $1.19 \pm$ \\
\hline$\%$ Change & & $0.04^{*}$ & $0.07 * *$ & $0.09 *$ \\
\hline
\end{tabular}

n.s. denoted to nonsignifcant, *Significant at $\mathrm{P}<0.05$, ** high significant at $\mathrm{P}<0.01$, ***very highly significant at $\mathrm{P}<0.001$ respectively. 
Table (9):Effects of candesartan( $3 \mathrm{mg} / \mathrm{kg}$ body weight), garlic $(100 \mathrm{mg} / \mathrm{kg})$ and their combination on serum enzyme levels compared with cholesterol diet (HCD) treated male mice. Mean \pm S.E. of five animals.

\begin{tabular}{|c|c|c|c|c|}
\hline Parameters & HCD & Candesartan & Garlic & $\begin{array}{l}\text { Candesartan } \\
\text { + Garlic }\end{array}$ \\
\hline $\begin{array}{l}\text { ALP (U/L) } \\
(\text { Mean } \pm \text { S.E.) }\end{array}$ & $\begin{array}{l}53.4 \pm \\
1.92\end{array}$ & $\begin{array}{l}115.24 \pm \\
0.28 * * *\end{array}$ & $\begin{array}{l}51.64 \pm \\
1.46^{\text {n.s. }}\end{array}$ & $87.8 \pm 4.3^{* * *}$ \\
\hline \% Change & & $+115.7 \%$ & $-3.3 \%$ & $+64.4 \%$ \\
\hline $\begin{array}{l}\text { AST }(\mathrm{U} / \mathrm{ml}) \\
(\text { Mean } \pm \text { S.E) }\end{array}$ & $\begin{array}{l}100.1 \pm \\
2.31\end{array}$ & $\begin{array}{l}150.7 \pm \\
6.11 * * *\end{array}$ & $\begin{array}{l}79.70 \pm \\
5.32^{* *} .\end{array}$ & $126.6 \pm 9.1 * *$ \\
\hline \% Change & & $+50.5 \%$ & $-20.4 \%$ & $+25.9 \%$ \\
\hline $\begin{array}{l}\text { ALT (U/ml) } \\
(\text { Mean } \pm \text { S.E) }\end{array}$ & $\begin{array}{l}66.32 \pm \\
2.54\end{array}$ & $\begin{array}{l}119.8 \pm \\
1.36^{* * * *}\end{array}$ & $\begin{array}{l}59.86 \pm \\
1.55^{* *}\end{array}$ & $\begin{array}{l}113.40 \pm \\
2.93 * * *\end{array}$ \\
\hline$\%$ Change & & $+80.0 \%$ & $-9.7 \%$ & $+70.9 \%$ \\
\hline $\begin{array}{l}\text { AChE (U/L) } \\
(\text { Mean } \pm \text { S.E.) }\end{array}$ & $359.0 \pm 19.77$ & $\begin{array}{r}467.0 \pm \\
22.18^{* *}\end{array}$ & $\begin{array}{l}126.3 \pm \\
4.56^{* * * *}\end{array}$ & $330.2 \pm 36.6^{\text {n.s. }}$ \\
\hline \% Change & & $+30.1 \%$ & $-64.8 \%$ & $-8.0 \%$ \\
\hline
\end{tabular}

n.s. denoted to nonsignificant, *Significant at $\mathrm{P}<0.05$, ** high significant at $\mathrm{P}<0.01$, $* * *$ very highly significant at $\mathrm{P}<0.001$ respectively.

Table (10): Effects of candesartan( $3 \mathrm{mg} / \mathrm{kg}$ body weight), garlic $(100 \mathrm{mg} / \mathrm{kg})$ and their combination on liver enzyme levels compared with cholesterol diet (HCD) treated male mice. Mean \pm S.E. of five animals.

\begin{tabular}{|c|c|c|c|c|}
\hline Parameters & HCD & Candesartan & Garlic & $\begin{array}{l}\text { Candesartan } \\
\text { + Garlic }\end{array}$ \\
\hline $\begin{array}{l}\text { ALP (U/L) } \\
(\text { Mean } \pm \text { S.E. })\end{array}$ & $\begin{array}{l}12.94 \pm \\
0.45\end{array}$ & $\begin{array}{l}15.44 \pm \\
0.295^{* * *}\end{array}$ & $\begin{array}{l}11.44 \pm \\
0.42 *\end{array}$ & $\begin{array}{c}13.8 \pm \\
0.58^{\text {n.s. }}\end{array}$ \\
\hline$\%$ Change & & $+19.3 \%$ & $-11.6 \%$ & $+6.6 \%$ \\
\hline $\begin{array}{l}\text { AST (U/ml) } \\
(\text { Mean } \pm \text { S.E) }\end{array}$ & $51.98 \pm 2.36$ & $\begin{array}{l}91.66 \pm \\
0.93 * * *\end{array}$ & $\begin{array}{l}50.20 \pm \\
1.82^{\text {n.s. }}\end{array}$ & $\begin{array}{l}33.8 \pm \\
1.9 * * *\end{array}$ \\
\hline \% Change & & $+76.3 \%$ & $-3.4 \%$ & $-34.9 \%$ \\
\hline $\begin{array}{l}\text { ALT (U/ml) } \\
(\text { Mean } \pm \text { S.E) }\end{array}$ & $15.32 \pm 0.29$ & $\begin{array}{l}18.64 \pm \\
1.04 * *\end{array}$ & $\begin{array}{l}12.80 \pm \\
0.49 * *\end{array}$ & $\begin{array}{c}11.02 \pm \\
0.32 * * *\end{array}$ \\
\hline$\%$ Change & & $+20.5 \%$ & $-16.4 \%$ & $-28.1 \%$ \\
\hline $\begin{array}{l}\text { AChE (U/L) } \\
\text { (Mean } \pm \text { S.E.) }\end{array}$ & $45.56 \pm 1.58$ & $\begin{array}{l}52.60 \pm \\
1.66 * *\end{array}$ & $\begin{array}{l}40.2 \pm \\
2.35^{\text {n.s. }}\end{array}$ & $\begin{array}{c}29.40 \pm \\
0.93 * * *\end{array}$ \\
\hline$\%$ Change & & $+15.2 \%$ & $-11.8 \%$ & $-35.5 \%$ \\
\hline
\end{tabular}

n.s. denoted to nonsignificant, *Significant at $\mathbf{P}<0.05, * *$ high significant at $\mathrm{P}<0.01$, ***very highly significant at $\mathbf{P}<0.001$ respectively. 
Fig .(1):plasma levels and tissue cholesterol content and plasma testosterone of ND and HCD of male mice.

$\square$ ND $\square \mathrm{HCD}$

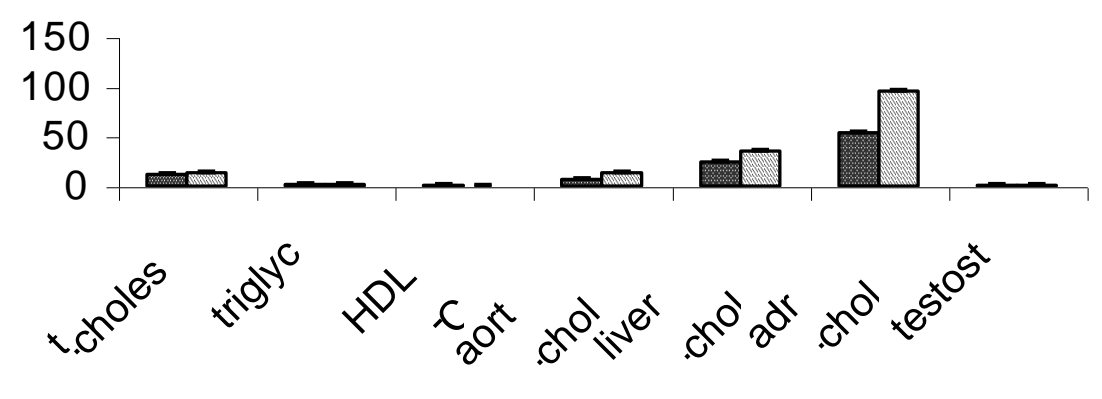

Fig .(2 a): Total WBCs, neutrophils and platelets of ND and HCD male mice .

$\square \mathrm{ND} \square \mathrm{HCD}$

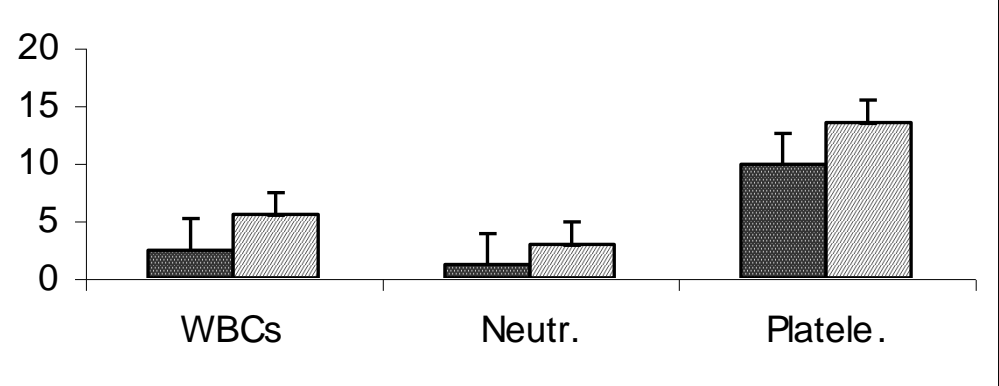

Fig .(2 b): Serum enzym levels of ND and HCD male mice.

$$
\text { 品D }
$$

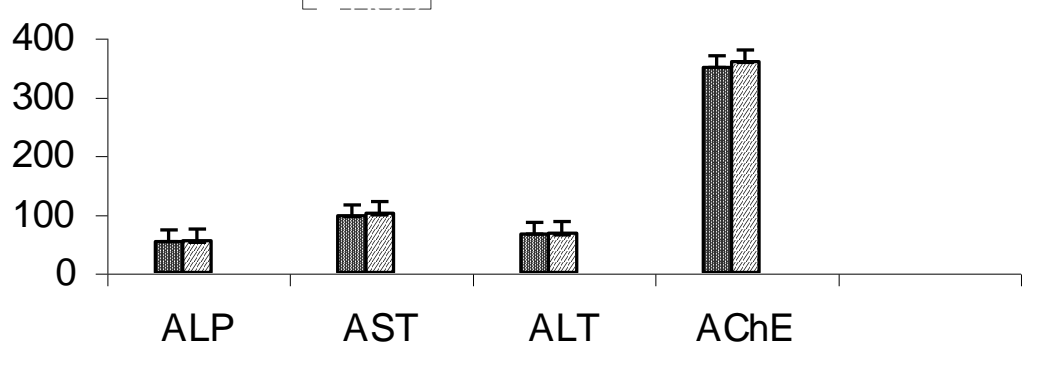


Fig.(3): Heart and body weights and liver enzymes of ND and HCD male mice .

$$
\square \mathrm{ND} \square \mathrm{HCD}
$$
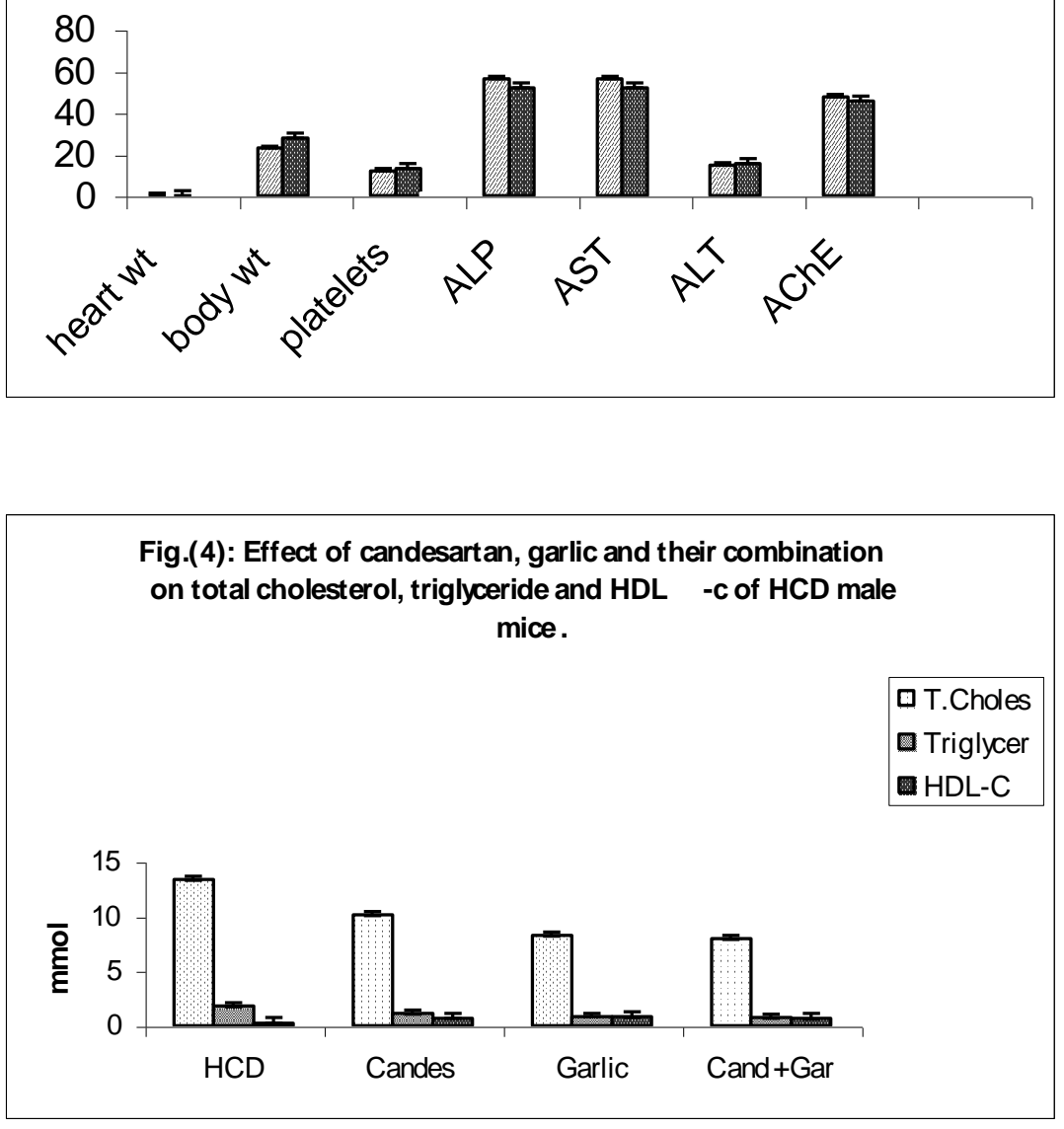

Fig.(5): Effect of candesartan, garlic and their combination on tissue cholesterol content $c$ HCD male mice.

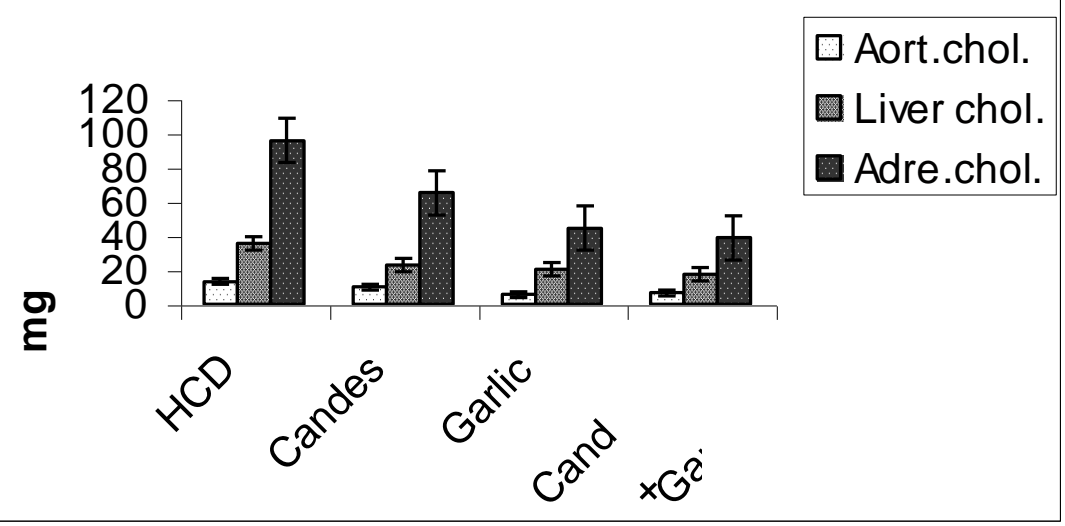




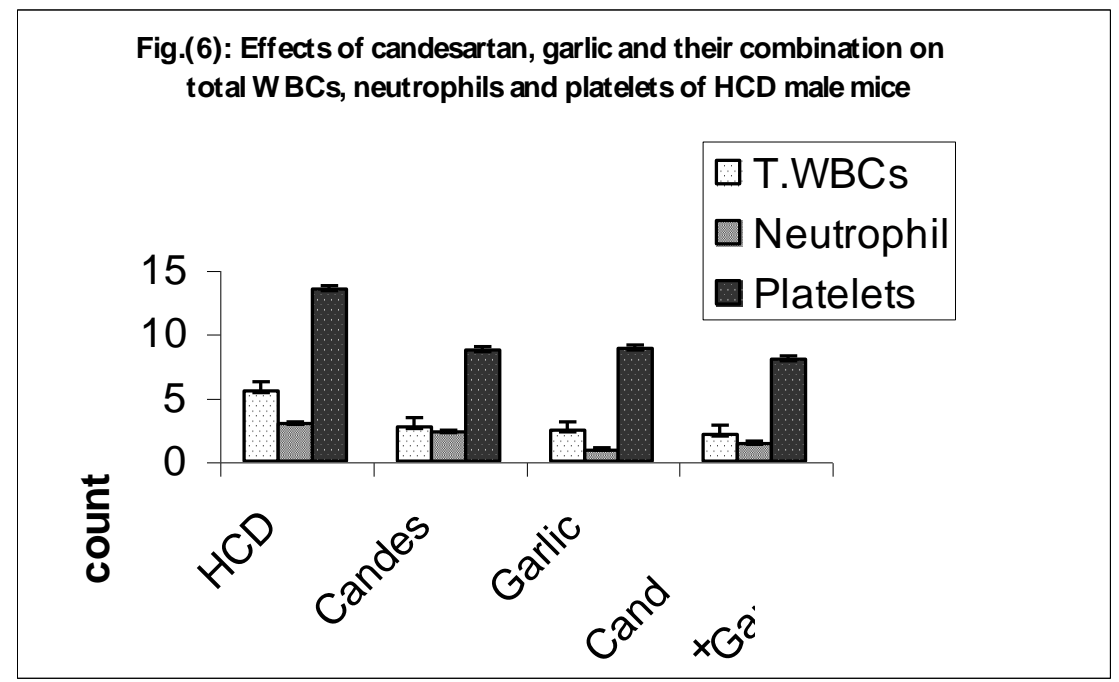

Fig.(7): Effect of candesartan, garlic and their combination on the heart and body weights of HCD male mice .

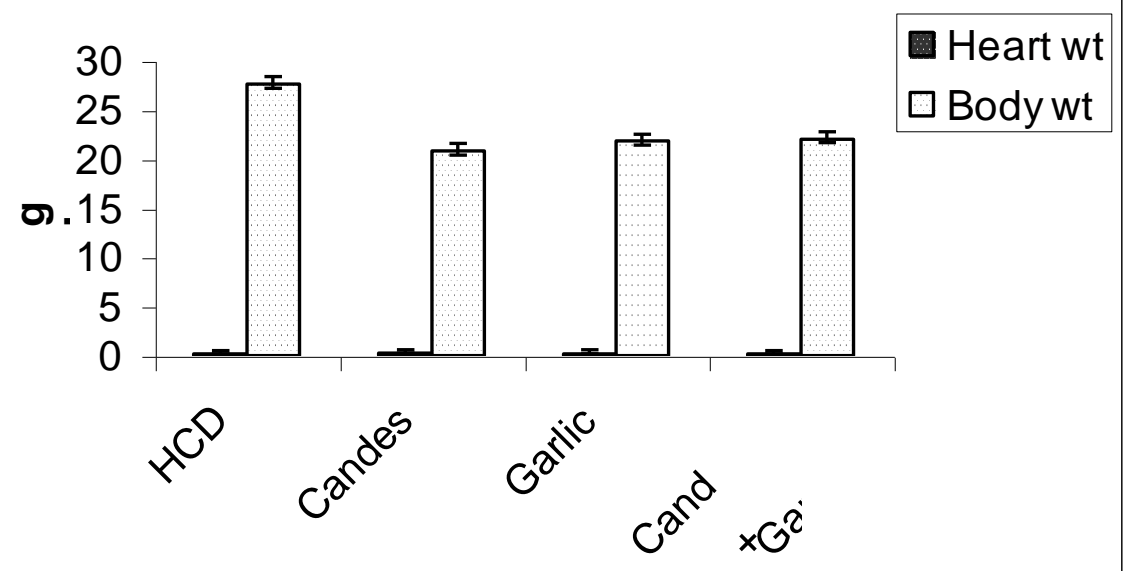

Fig.(8): Effect of candesartan, garlic and their combination on plasma testosterone of HCD male mice

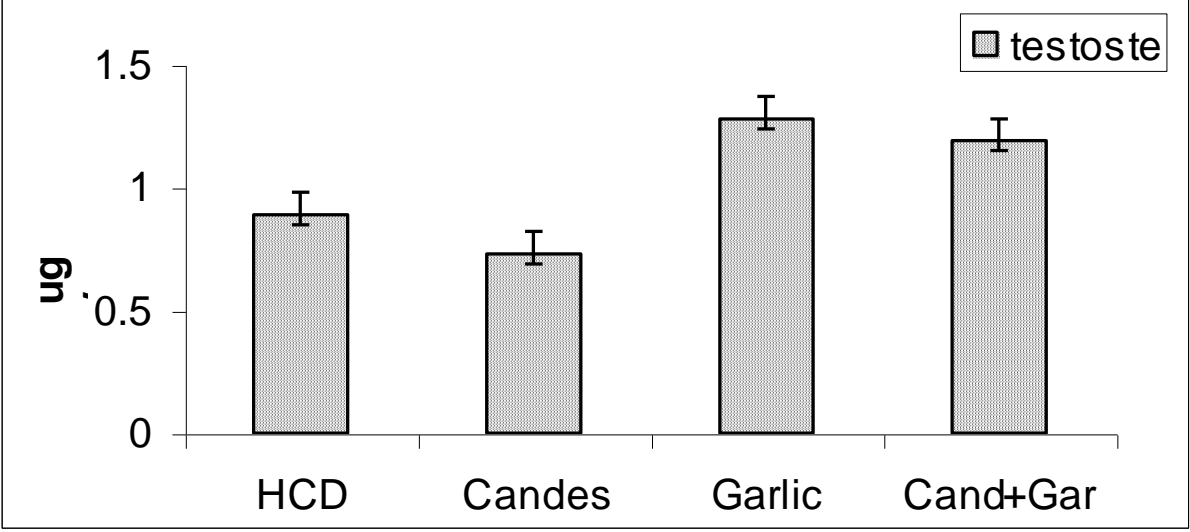



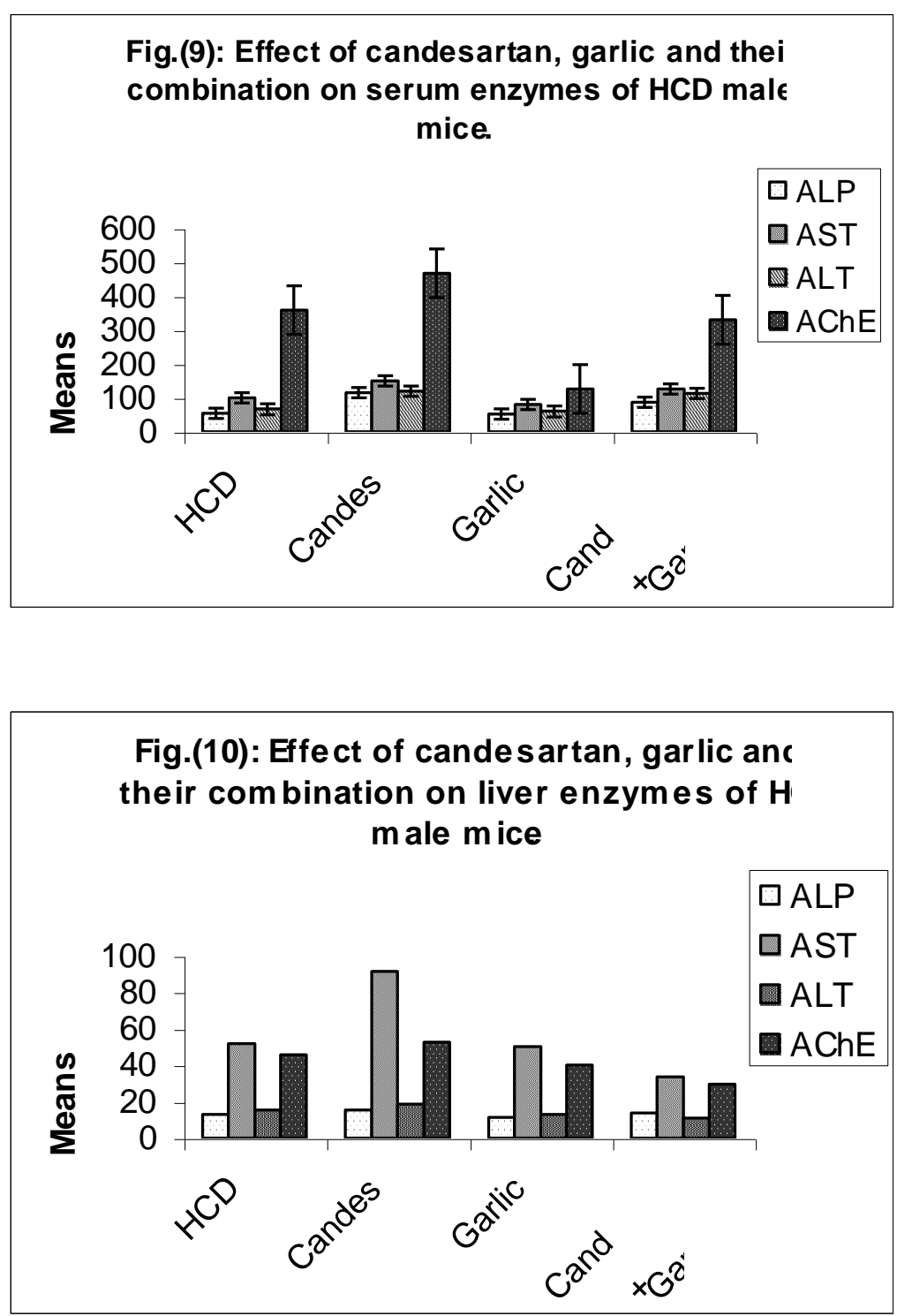

\section{Discussion}

Elevated serum cholesterol has long been associated with an risk for coronary artery disease and the development of myocardial infarction (Wesley et al.,1999). The effects of hypercholesterolemia on atherosclerosis and vascular function have been extensively studied by number of investigators. One of the earliest and most easily detected events associated with elevated cholesterol levels is a diminution in nitric oxide release by vascular endothelium (Lefer and Ma,1993). Impaired endothelium dependent vascular reactivity has been reported in a number of blood vessel type and a variety of animal models of hypercholesterolemia (Cooke et al.,1991 and Rossitch et al.,1991). In addition, endothelium-dependent vascular reactivity is impaired in forearm (Gilligan et al.,1994) and coronary microcirculation (Drexler et al.,1991) of human. The endothelial cell dysfunction resultant from an high cholesterol diet (HCD) has been shown to promote interactions between circulating leukocytes and endothelium (Gauthier et al.,1995). Enhanced vascular immune responses to hypercholesterolemia are considered a hallmark of this condition 
and it is now believed that hypercholesterolemia induces an inflammatory condition (Berliner et al.,1995). This response to hypercholesterolemia is though to be pivotal step in the development of atherosclerotic lesions and has been shown to be effectively reversed by treatment with $\mathrm{NO}$ therapy or with the NO precursor Larginine ( Drexler et al.,1991 and Gauthier et al.,1995). Enhanced vascular immune responses to hypercholesterolemia are considered a hallmark of this condition and it is now believed that hypercholesterolemia induces an inflammatory condition (Berliner et al.,1995 ; Libby and Hansson,1991). Along these lines, it has been reported that hypercholesterolemic rats exhibit enhanced leukocyte adhesion and microvascular protein leakage in the setting of mesenteric ischemia reperfusion (Kurose et al.,1997). The observed inhibitory effect of candesartan and garlic on atherosclerosis development in the present study is similar to an earlier report (Keidar et al.,1997). The observed inhibitory effect of candesartan and garlic on atherosclerosis development may explain on the basis that $\alpha 1$-adrenergic blocker decrease LDL level via increasing receptor -mediated catabolism of LDL as well as increasing HDL level(Pool et $a l ., 1990)$. The most interesting and important findings of this investigation were the prevention of atherosclerosis and cholesterol accumulation in candesartan cilexetil and garlic treated animals. In a private consulting practice setting, the addition of candesartan in normotensive patients with chronic renal disease and proteinuria receiving angiotensin converting enzyme inhibitor reduced proteinuria and blood pressure ( Smith et al.,2003). The angiotensin II receptor blocker ( andesartan cilexetil) demonstrated similar efficacy in lowering blood pressure with significant swelling in patient "feet and ankles compared to the most prescribed antihypertensive medication (Weinberger, 2000). Cardiovascular studies have been mainly related to atherosclerosis, where effects were examined on serum cholesterol, LDL, HDL and triglycerides. Lowering of serum lipids by garlic ingestion may decrease the atherosclerosis process. Allicin and adenosine are the most potent antiplatelet constituents of garlic because of their in vitro effect (Agarwal,1996). In that study, mice were subjected to 12 weeks of an high cholesterol diet (HCD) and then exposed to various stimuli, including candesartan cilexetil, garlic and their combination. The previous results are somewhat agreed to our observations of inflammation caused by hypercholesterolemia and its relation with leukocytes and platelets. It could be concluded that, in spite of the presence of some differences in the effect of candesartan, garlic each alone and in combination on male testosterone, there is some correlation between capability of candesartan to reduce the total cholesterol and the process of steroidogenesis. As well garlic induced elevation of testosterone in spite of its lowering to cholesterol levels.

In a more generalized discussion, Harper et al.(1979) indicated that serum transaminases extensive tissue damage these enzymes are liberated into serum; they mentioned as examples the rise of both enzymes in serum in cases of myocardial infarction. The above results may be considered as an indication to myocardial or hepatic damage or some other biochemical lesions resulting in an increase in the titre of these enzymes in the serum. These results agree with report of Sheets et al.(1977). The enzymes ALP,AST, ALT and $\mathrm{ChE}$ are not normally functional in the serum. Their presence is due to their release from organs and tissues rich in them, such as the liver, brain, myocardium, bones and skeletal muscles. Hence the increase in serum levels of these enzymes following the administration of the candesartan, reflects their release from these tissues as result of tissue injury or increased membrane permeability.

\section{References}

1. Agarwal,K.C.(1996): Therapeutic action of garlic constituents. Med. Res. Rev.,16(1):111-124.

2. Banerjee, S.K. and Mualik, S.K.(2002): Effect of garlic on cardiovascular disorder. Nutr. J.,19(1):1-4. 
3. Belfeild, A. and Goldberg. M.D. (1971): Revised assay for serum phenyl phosphatase activity using 4-amino antipyrine. Enzyme (12): 561.

4. Berliner,J.A.; Navab,M.; Fogelman, A.M.; Frank, J.S.; Demer, L.L.; Edwards, P.A.; Watson, A.D. and Lusis, A.J.(1995): Atherosclerosis: basic mechanisms: oxidation inflammation and genetics. Crculation.,91: 2488-2496.

5. Berthold,H.K.; udhop,T.; Bergmann,K. V. (1998): Effect of garlic oil preparation on serum lipoproteins and cholesterol metabolism. JAMA, 279:1900-1902.

6. Buccolo, G. and David, H.(1973): Quantitative determination of serum Triglycerides by the use of enzymes. Clin. Chem.; 36: 476-482.

7. Chobanian, A.V. and Alexander, R.W.(1996): Exacerbation of atherosclerosis by hypertension. Potential mechanisms and clinical implications. Arch. Intern. Med., 156:1952-1956.

8. Cooke,J. P., Andon, N.A.; Girerd,X.J.; Hirsch, A.T. and Creager, M.A.(1991): Arginine restores cholinergic relaxation of hypercholesterolemic rabbit thoracic aorta. Circulation. 38:1057-1062.

9. Doyle, A.E. (1991): Does hypertension predispose to coronary artery disease? In hypertension: Pathophysiology, diagnosis and management. Laragh J.H., Brenner B.M., editors, New York, NY: Raven Press Ltd., P119-125.

10. Drexler, H.; Zeiher, A.M.; Meinzer, K.; Just, H.(1991): Correction of endothelial dysfunction in coronary microcirculation of hypercholesterolemic patient by Largenine. Lancet., 338:1546-1550.

11. Elman, G.L.; Courtney, K.D.; Endres, G. Jr. and Featherflone, R.M. (1979): A new an rapid colourimetric determination of acetylcholinestrase activity. Biochem. Pharmacol., 7:88-93.

12. Fallon, M.B.; Abrams, G.A.;Abdel Razek,T.T.;Dai,J.;Oparil,S. and Ku,D.D.(1998): Garlic prevents hypoxic pulmonary hypertension in rats. Am. J. Physiol. Lung Cell Mol Physiol.,275:L283L287.

13. Gauthier,T.W.;Scalia,R.;Murohara,T.;G ua,J.P.and Lefer, A.M.(1995): Nitric oxide protects against leukocyteendothelium interactions in the early stages of hypercholesterolemia. Arterioscler Thromb Vasc Biol.,15:1652-1659.

14. Gilligan,D.M.; Guetta, b.; Panza, J.; Garcia, C.E.; Quyyumi, A.A. and
Cannon, R.O.(1994): Selective loss of microvascular endothelial function in human hypercholesterolemia. Circulation., 90: 35-41.

15. Golino,P.; Maroko, P.R. and Carew, T.E. (1987): Efficacy of platelet depletion in counteracting the detrimental effect of acute hypercholesterolemia on infarct size and no-reflow phenomenon in rabbits undergoing coronary artery occlusionreperfusion. Circulation,76:173-180.

16. Harper, A.H.; Rodwell, V.W. and Mayes, P.A.(1979): Review of physiological chemistry $17^{\text {th }}$ ed.,702.pp. Lange Medical Publications, Los Altos, California, USA.

17. Hawk, P.B.; Oser,B.L. and Summerson, W.(1965):Hawk's hysiological Chemistry. $12^{\text {th }}$ ed .,G.A. Churchill Ltd ,London.

18. Kannel, W.B.;Castelli, W.P.; Gordon, T and McNamara ,P.(1971): Serum cholesterol, lipoproteins and the resk of coronary heart disease: The Framingham Study. Ann. Intern. Med., 74:1-2.

19. Katz,R.I.and Kopin,I.J.(1969): Electrical field stimulation release norepinephrin from rat atrium: effects of ions and drugs. J.Pharmacol. Exp. Ther.,169: 229-236.

20. Keidar,S.; Attias, J.; Smith, J.; Breslow, J.L. and Hayek, T. (1997): The angiotensin-II receptor antagonist, losartan, inhibits LDL lipid peroxidation and atherosclerosisin apolipoprotein E-deficient mice. Biochem Biophys Res Commun, 236:622-627.

21. Kirpekar,S.M. and Misu, Y.(1967): Release of noradrenaline by splenic nerve stimulation and its dependence on calcium. J. Physiol. (London). 188:219-234.

22. Kurose, I.;Argenbright, L.W.; Anderson, D.C.; Tolley,J.; Miyasaka, M. and Granger, D.N.(1997): Reperfusioninduced leukocyte adhesion and vascular protein leakage in normal and hypercholesterolemic rats. AM J Physiol., 273: H854-H860.

23. Lefer, A.M. and Ma, X.L.(1993): Decreased basal nitric oxide release in hypercholesterolemia increases neutrophil adherence to rabbit coronary artery endothelium. Arterioscler. Thromb.,13: 771-776.

24. Libby, P.and Hansson, G.K.(1991): Involvement of the immune system in human atherogenesis:current knowledge and unanswered questions. Lab Invest., 64: 4-14.

25. Moran, M. (2000): Adding candesartan to angiotensin converting enzyme inhibitor in 
renal disease. Nephron Dial Transplant., 17: 597-601.

26. Neal, B. and MacMahons, S.(1999): The world health organization-international socity of hypertension blood pressure lowering treatment trialists-collaboration: prospective collaborative overviews of major randomized trials of blood pressurelowering treatments. Curr-Hypertens. Rep., 1:346.-356.

27. Osborne, J.A.; Mentley, R.K.and Lefer, A.M.(1987): Increased severity of acute myocardial ischemia in experimental atherosclerosis. Heart Vessels,3:73-79.

28. Osborne,J.A.; Lento, M.R.; Siegfried, M.R.; Stahl, G.L., Fusman, B. and Lefer, A.M.(1989): Cardiovascular effects of acute hypercholesterolemia in rabbits: reversal with lovastatin treatment. J Clin Invest., 83: 465-473.

29. Orekhov, A.N. and Grunwald, J.(1997): Effects of garlic on atherosclerosis. Nutr., 13(78):656-663.

30. Pool, J.L.; Lenz, M.L. and Taylor, A.A. (1990): Alpha 1-adrenoreceptor blockade and the molecular basis of lipid metabolism alteration. J Hum Hypertens, 4:23-33.

31. Raasch, W.; Bartels, T.; Gieselberg, A.; Dendorfer, A. and Dominiak, P. (2002): Angiotensin I -Converting Enzyme inhibition increases cardiac catecholamine content and reduces Monoamine Oxidease Activity via an Angiotensin Type 1 Receptor- Mediated Mechanism. Pharmacology and Exp. Therapeutics, 300 (2): $248-4340$

32. Reitman, S. and Frankel, S. (1975): A colourimetric method for determination of serum glutamic oxaloacetate and glutamic pyruvic transaminase. Amer. J. Clin. Path., 28: 26-34.

33. Rossitch, E.; Alexander,E.; Black, P. M. AND Cooke, J.P.(1991): L-Arginine normalizes endothelial function in cerebral vessels from hypercholesterolemic rabbit. J Clin Invest., 87:1295-1299.

34. Rubin,R.P.(19700: The role of calcium in the realease of neurotransmitter substances and hormones. Pharmacological Review,22(3):389-428.

35. Sheets, L.P.; Hamilton, B.F.; Sangha,G.K. and Thyssen, J.H.(1977): Subchronic neurotoxicity screening studies with organophosphate and morphology relative to cholinesterase inhibition. Fundamental and Appl. Toxicol., 35(1):101-119.
36. Smith, K.P.; Kenneth, F. and David, P. (2003): Randomized controlled crossover study of the effect on proteinuria and blood pressure of adding an angiotensin II receptor antagonist toan angiotensin converting enzyme inhibitor in the normotensive patients with chronic renal disease and proteinuria. Nephrol Dial Transplant, 17: 597-601.

37. Snedecor,G.W. and Cochran, W. (1969): Statistical methods, $6^{\text {th }}$ ed. The Iowa state U.S.A.

38. Spigelski, D. and Jones, P.J.(2001): Efficacy of garlic supplementation in lowering serum cholesterol levels. Nutr.Rev.,59(7):236-241.

39. Takishita, S.(2001): Antihypertensive drug therapy: Adverse effects and drug interactions. Nippon Kinsho, 59: 992-997.

40. Tilton,R.G.; Cole, P.A.; Zions, J.D.; Daugherty,A.;Larson,K.;Sutera,S.P.; ilo,C. and Williamson, J.K.(1987): Increased ischemia-reperfusion injury to the heart associated with short term, diet induced hypercholesterolemia in rabbits. Circ. Res., 60:551-559.

41. Tsao, P.S.; McEvoy, L.M.; Drexler, H.; Butcher, E.C. and Cooke, J.P. (1994): Enhanced endothelial adhesiveness in hypercholesterolemia is attenuated by Larginine. Circulation. 89: 2176-2182.

42. Velez de la call, J.F.; Soufir, J.C.; Chodarge, F.; Boisseau, C.; Kercret, $\mathbf{H}$. and Zegou, B. (1988): Reproductive effects of anticancer drug procarbazine in male rat at different ages. J. Reprod. Fertil., 84: 51-61.

43. Warnick, G.R.; Benderson, V. and Albers, N. (1983): Selected methods. Clin. Chem.; 10: 91-99.

44. Weber, M.A.; David, H.G.; Smith, D.H.; Neutel, J.M. and Graettinger, W.F.(1991); Cardiovascular and metabolic characteristics of hyprertension. Am.J. Med. ,91:5 S-10 S.

45. Weinberger, M.H. (2000):ASH: Atacand (Candesartan) Effective Antihypertensive, More Tolerable Than Norvasc (Amlodipine). University School of Medicine and a Castle Study ivestigator. New York, NY -Abstract.

46. Wesley, G.G.; Steven, P.J.; Nola Sieber; Tak, Y.A. and David, J.L.(1999); Effects of Hypercholesterolemia on Myocardial Ischemia-Reperfusion Injury in LDL Receptor Deficient Mice. Atherosclerosis, Throbosis and Vascular Biology, 19:2776. 


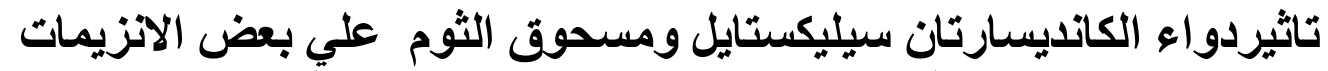

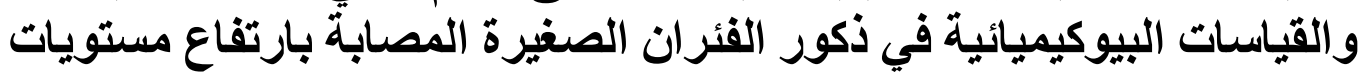 الاهون
}

\author{
د/ محمود رابح محمود \\ قسم علم الحيوان - كلية العلوم - جامعة الزقازيق - جمهورية مصرد
}

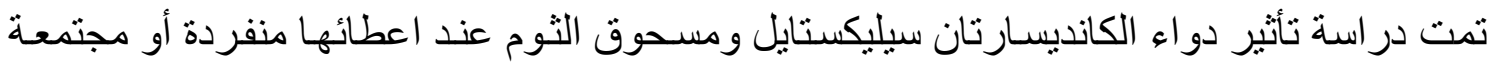

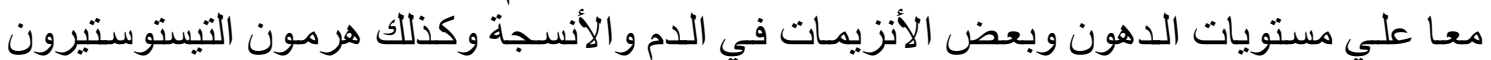

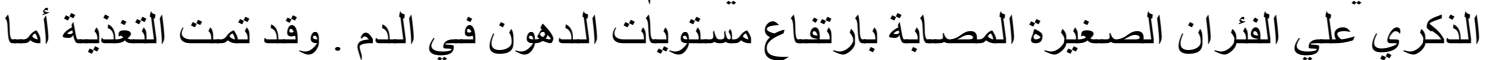

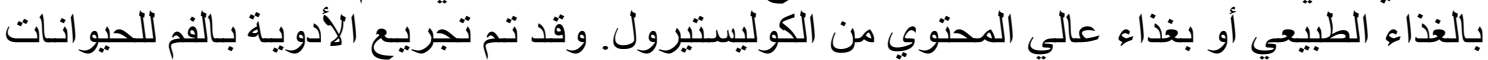

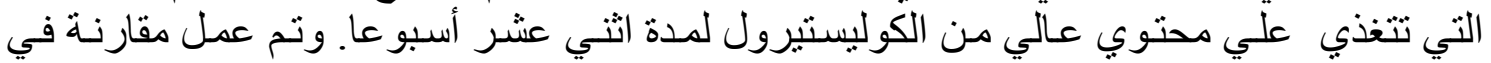

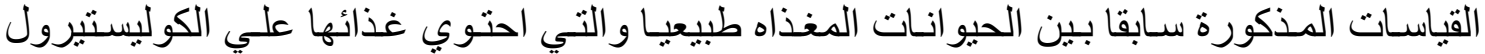

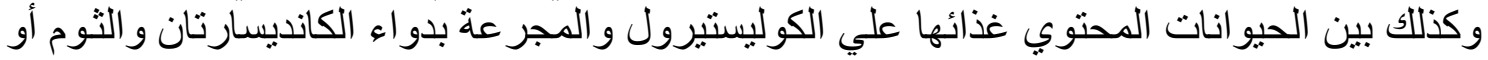

مجتمعة معا.

و أظهرت النتائج أن الحيو انات التي يحتوي غذائها علي الكوليستيرول بنسبة عاليـة سجلت أرتفاع

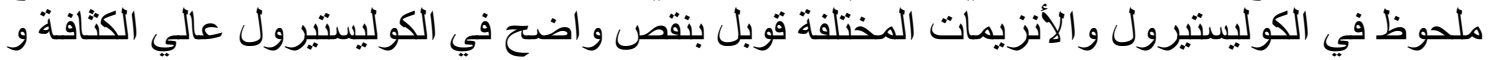

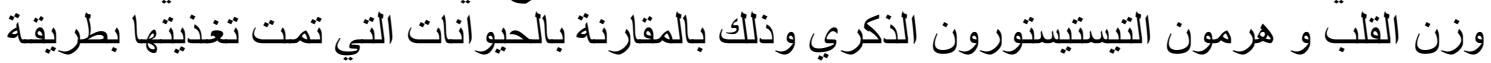
عادية.

و علي الجانب الآخر وبالمقارنـة بتأثنير الكانديسارتان و الثوم منفردين أو مجتمعين علي الحيو انـات

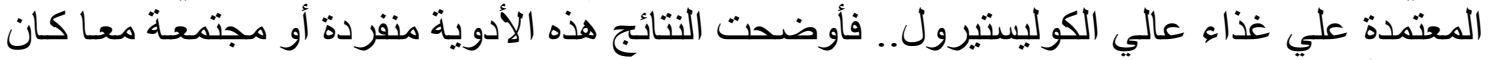

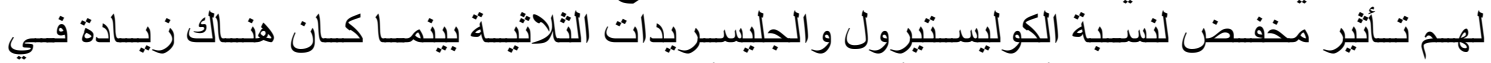

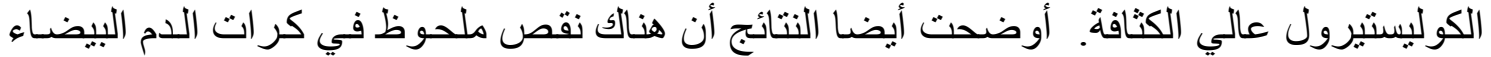

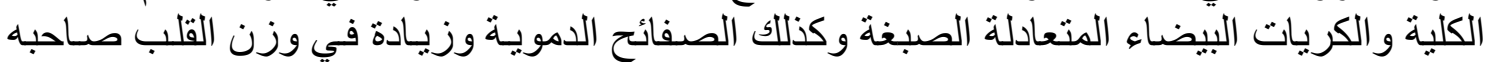

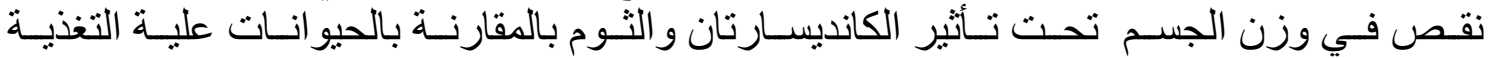

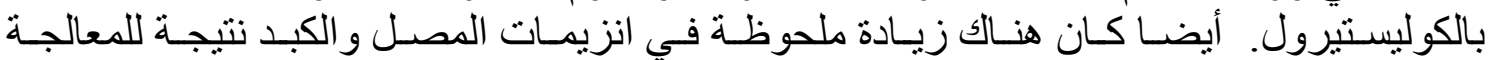

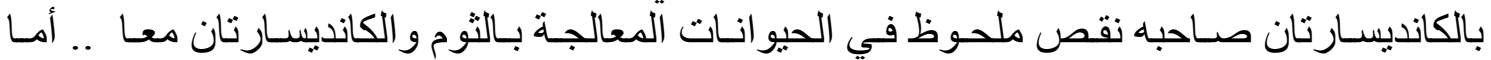

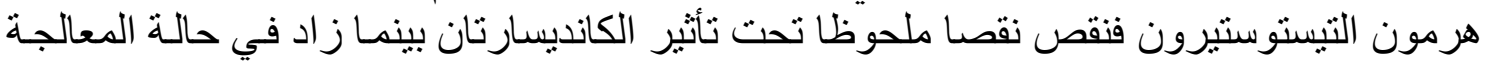
بمسحوق الثوم.. و الثوم و الكانديسارتنان معا.

و الخلاصة أنه بالر غم من قدرة الكانديسارتان والثوم علي خفض الكوليستيرول فأن تأثير الثوم أحدث

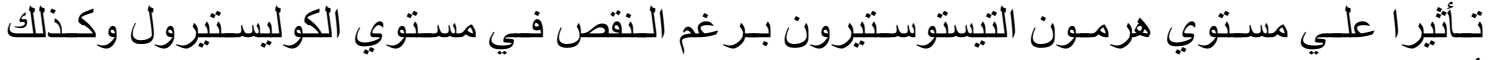

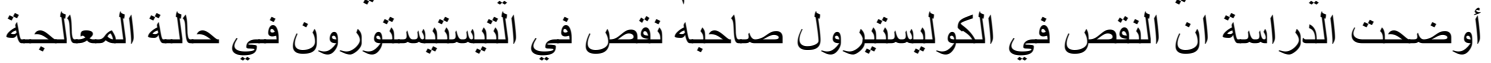

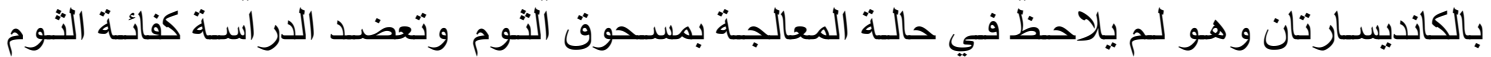
كركب و اق في تخفيف بعض الأضرار الخلوية الناتجة بو اسطة المعالجة بالكانديسارنان. 